\title{
Mesoporous polydopamine nanoparticles carrying peptide RL-QN15 show potential for skin wound therapy
}

Pan Qin ${ }^{4 \dagger}$, Yi Meng ${ }^{1+}$, Ying Yang ${ }^{5 \dagger}$, Xinyu Gou ${ }^{2 \dagger}$, Naixin Liu' ${ }^{1}$, Saige Yin ${ }^{1}$, Yan Hu ${ }^{1}$, Huiling Sun ${ }^{1}$, Zhe Fu ${ }^{1}$, Yinglei Wang ${ }^{1}$, Xiaojie Li ${ }^{4}$, Jing Tang ${ }^{4}$, Ying Wang ${ }^{3^{*}}$, Ziwei Deng ${ }^{2^{*}}$ and Xinwang Yang ${ }^{1 *}$ (D)

\begin{abstract}
Background: Skin wound healing remains a considerable clinical challenge, thus stressing the urgent need for the development of new interventions to promote repair. Recent researches indicate that both peptides and nanoparticles may be potential therapies for the treatment of skin wounds.

Methods: In the current study, the mesoporous polydopamine (MPDA) nanoparticles were prepared and the peptide RL-QN15 that was previously identified from amphibian skin secretions and exhibited significant potential as a novel prohealing agent was successfully loaded onto the MPDA nanoparticles, which was confirmed by results of analysis of scanning electron microscopy and fourier transform infrared spectroscopy. The encapsulation efficiency and sustained release rate of RL-QN15 from the nanocomposites were determined. The prohealing potency of nanocomposites were evaluated by full-thickness injured wounds in both mice and swine and burn wounds in mice.

Results: Our results indicated that, compared with RL-QN15 alone, the prohealing potency of nanocomposites of MPDA and RL-QN15 in the full-thickness injured wounds and burn wounds in mice was increased by up to 50 times through the slow release of RL-QN15. Moreover, the load on the MPDA obviously increased the prohealing activities of RL-QN15 in full-thickness injured wounds in swine. In addition, the obvious increase in the prohealing potency of nanocomposites of MPDA and RL-QN15 was also proved by the results from histological analysis.
\end{abstract}

\footnotetext{
*Correspondence: wangying_814@163.com; zwdeng@snnu.edu.cn; yangxinwanghp@163.com

${ }^{\dagger}$ Pan Qin, Yi Meng, Ying Yang and Xinyu Gou contributed equally to this work

${ }^{1}$ Department of Anatomy and Histology and Embryology, Faculty of Basic Medical Science, Kunming Medical University, Kunming, Yunnan 650500, China

${ }^{2}$ Key Laboratory of Applied Surface and Colloid Chemistry, National Ministry of Education, Shaanxi Key Laboratory for Advanced Energy Devices, Shaanxi Engineering Lab for Advanced Energy Technology, School of Materials Science and Engineering, Shaanxi Normal University, Xi'an, Shaanxi 710119, China

${ }^{3}$ Key Laboratory of Chemistry in Ethnic Medicine Resource, State Ethnic Affairs Commission and Ministry of Education, School of Ethno-Medicine and Ethno-Pharmacy, Yunnan Minzu University, Kunming, Yunnan 650504 , China

Full list of author information is available at the end of the article
} permits use, sharing, adaptation, distribution and reproduction in any medium or format, as long as you give appropriate credit to the original author(s) and the source, provide a link to the Creative Commons licence, and indicate if changes were made. The images or other third party material in this article are included in the article's Creative Commons licence, unless indicated otherwise in a credit line to the material. If material is not included in the article's Creative Commons licence and your intended use is not permitted by statutory regulation or exceeds the permitted use, you will need to obtain permission directly from the copyright holder. To view a copy of this licence, visit http://creativecommons.org/licenses/by/4.0/. The Creative Commons Public Domain Dedication waiver (http://creativeco mmons.org/publicdomain/zero/1.0/) applies to the data made available in this article, unless otherwise stated in a credit line to the data. 
Conclusions: Based on our knowledge, this is the first research to report that the load of MPDA nanoparticles could significantly increase the prohealing potency of peptide and hence highlighted the promising potential of MPDA nanoparticles-carrying peptide RL-QN15 for skin wound therapy.

Keywords: Wound healing, Mesoporous polydopamine, RL-QN15, Nanoparticles

\section{Introduction}

Skin is exposed to many external and endogenous factors that can impact structure and function, making it easy to form wounds. Rapid repair after skin damage is an important precondition for protecting the internal organs from the external environment, which mainly consists of four stages: hemostasis, inflammatory response, cell proliferation and tissue reconstruction [1]. Physiological regulation of skin wound healing is a complex process that depends on interactions among many cell types and mediators in a highly sophisticated temporal sequence [2]. Therefore, it can be interrupted by many factors, resulting in non-healing chronic wounds or unhealing skin ulcer. An aging population coupled with escalating rates of diabetes and obesity continue to increase the prevalence of chronic wounds, leading to a sharp rise in medical costs [3]. Chronic wounds cause secondary infections, as well as water and electrolyte disturbance, infectious shock, multiple organ failure, and even death $[4,5]$. Thus, the integrity of skin structure and function is really crucial for human health and survival.

While extensive research has been conducted to identify new methods for improving skin wound healing, it still fails to meet clinical needs. Traditional wound healing drugs can be classified into two main groups: small molecule compounds derived from plants and proteins typified by epidermal growth factors (EGFs). Although these drugs can promote wound healing, there are still drawbacks; for example, the former compounds are unstable with relatively low activity, and the latter proteins are expensive, require rigorous preservation conditions, and are liable to cause hyperplastic scars [6]. In addition, stem cells from various sources are considered to be an attractive alternative method for wound repair [7-9]. However, the poor viability of stem cells in wound beds characterized by a harsh inflammatory environment often decreases the therapeutic potential of the cells $[10$, 11]. Thus, the development of novel promising interventions for the treatment of chronic skin wounds are definitely urgently awaited.

At present, nanomaterials have provided additional therapeutic strategies for wound repair. The main types of nanomaterials used for wound treatment are represented by nanoparticles, nanocomposites, hydrogels and scaffolds. Nanoparticles in treating wounds are mainly due to their intrinsic characteristics that aid wound closure or as delivery vectors for therapeutic agents. In particular, nano-drug delivery vectors can anchor bioactive molecules to an applied area, sustain drug release, and enhance the therapeutic efficacy of drugs, and are thus particularly relevant to the field of skin regeneration [12]. Mesoporous polydopamine (MPDA), as an excellent carrier featured by simple preparation protocol, strong adhesive properties, easy and straightforward functionalization, and biocompatibility, has the following preconditions: (1) The mesoporous structure of MPDA can be loaded into drug molecules; (2) MPDA contains many functional groups such as catechol, amine, and imine for chemical modification [13, 14]. In addition, MPDA nanoparticles were synthesized with uniform pores by a one-pot method without additional catalysts or toxic reagents, which has the advantages of simplicity, mildness, and environmental friendliness. However, the reports on the improvement of bioavailability fulfilled by MPDA nanoparticles still remain in their infancy. Previous report has revealed that the drug doxorubicin hydrochloride is encapsulated in the MPDA particles to kill the cancer cells and shows an unprecedentedly high payload [15]. However, the attempt to accelerate the regeneration of skin wounds afforded by the MPDA nanoparticles is still rarely reported.

In recent years, peptides have gradually attracted extensive interest due to their diverse functions and high activity, stability, and specificity [16-18]. Recent researches have shown that peptides from plants and animals have a wide range of bioactivities, e.g., antimicrobial, hyperuricemia-alleviating, pro-healing, and antioxidant activities [19-22]. We previously identified a short peptide (named RL-QN15, primary sequence 'QNSYADLWCQFHYMC') from the skin secretions of Rana limnocharis, which promoted the proliferation and migration of skin cells [23]. When at concentrations of nM levels, RL-QN15 significantly promote the healing of full-thickness injured wounds and diabetic skin chronic wounds in mice. However, we still hope that the ability of RL-QN15 to promote repair activity can be improved by some ways, so as to provide new strategies for the development of novel therapeutics for the treatment of wound healing which are still a harsh clinical challenge.

In the current research, we successfully prepared the MPDA nanoparticles and constructed the nanocomposites of MPDA and RL-QN15. The prohealing potency 


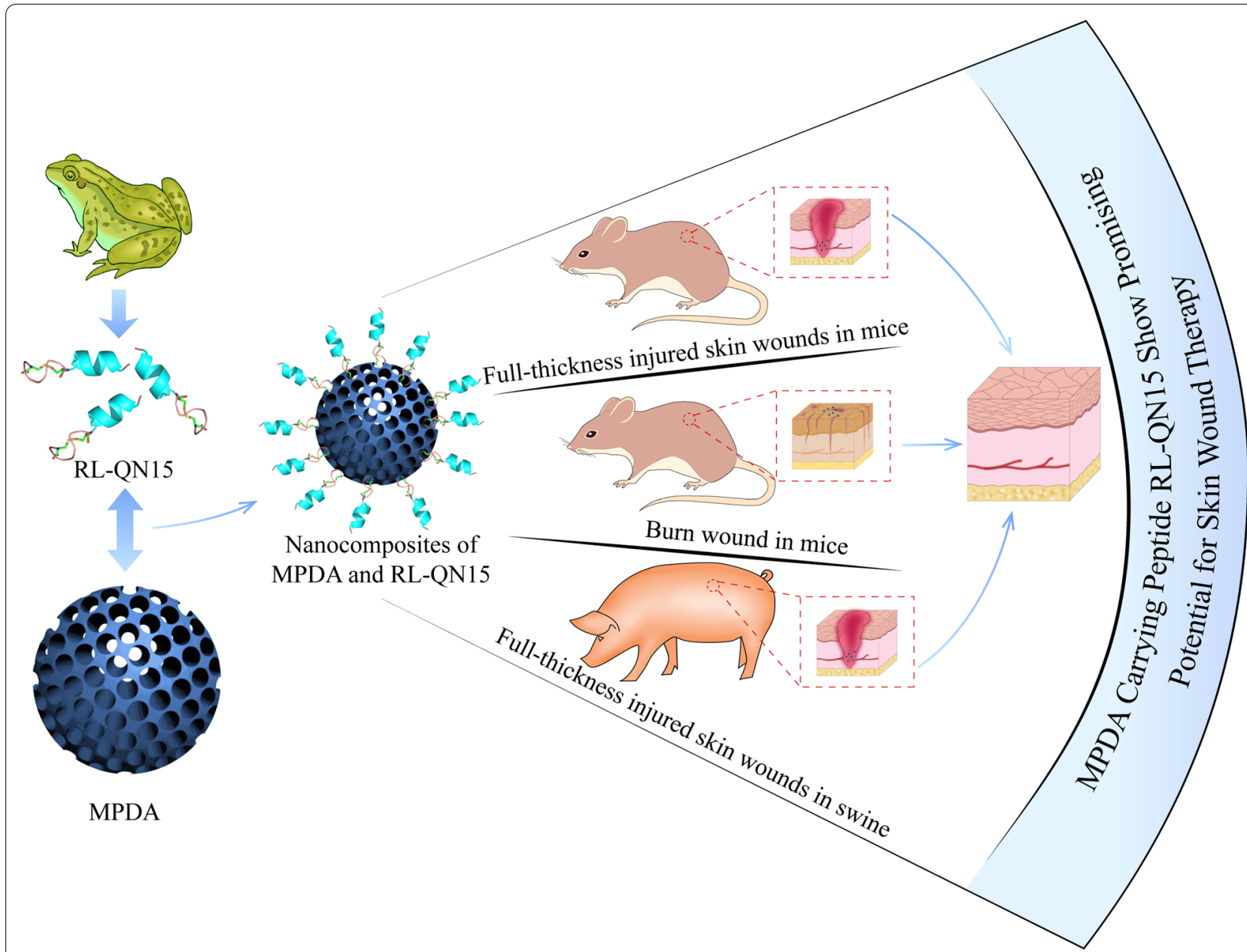

Scheme 1 Peptide RL-QN15 exerts increase skin wound healing potency by the load of MPDA

of RL-QN15 in full-thickness injured skin wounds (mice and swine) and burns (mice) was significantly enhanced by the load of MPDA nanoparticles. The synthesis process and application in different wound types of nanocomposites of MPDA and RL-QN15 are shown in Scheme 1. At present, some studies have shown that MPDA nanoparticle is an excellent delivery carrier for drugs to inhibit tumor growth [24]. Based on our knowledge, this is the first research to report that the load of MPDA nanoparticles could significantly increase the prohealing potency of peptide and hence highlighted the nanocomposites of MPDA and RL-QN15 as a promising intervention for the acceleration of skin wounds.

\section{Experimental section}

\section{Materials}

All chemical reagents were of analytical grade and used as received without further purification. Dopamine hydrochloride (DA, 98\%), triblock copolymer Pluronic F-127, and 1, 3, 5-trimethylbenzene (TMB) were purchased from Sigma-Aldrich (USA). Aqueous ammonia $\left(\mathrm{NH}_{3} \cdot \mathrm{H}_{2} \mathrm{O}, 25.0-27.0 \%\right)$ and ethanol were purchased from Sino-Pharm Chemical Reagent Co., Ltd (China). Peptide with purity higher than $95 \%$ used in this research were commercially synthesized by Wuhan Bioyeargene Biotechnology Co. Ltd. (Wuhan, China).

\section{Ethics statement}

All animal experimental procedures followed the Guide for the care and Use of Laboratory Animals and were approved by the animal ethics committee of Kunming Medical University, China (kmmu20211351). All surgeries were performed under anesthesia, and all efforts were made to minimize suffering.

\section{Synthesis of MPDA nanoparticles}

The MPDA particles were synthesized by a one-pot synthesis method according to previously reported procedures [25]. Typically, $0.15 \mathrm{~g}$ of dopamine and $0.10 \mathrm{~g}$ of triblock copolymer Pluronic F-127 were first dissolved 
in a mixture of water $(5 \mathrm{~mL})$ and ethanol $(5 \mathrm{~mL})$ under stirring at room temperature. Then, $0.16 \mathrm{~mL}$ of TMB was introduced to the mixture. After ultrasonication for 2 min in a water bath, the mixture gradually formed an emulsion solution. Subsequently, $0.375 \mathrm{~mL}$ of ammonia solution was quickly added to the reaction mixture under stirring. After $2 \mathrm{~h}$ reaction under room temperature, the resultant mesoporous PDA particles were collected by centrifugation and washed with ethanol and water for three times. Template removal was performed by extraction, where the as-prepared PDA particles were treated in a mixed solvent of ethanol and acetone $(\mathrm{v} / \mathrm{v}, 2 / 1)$ under ultrasonication for $30 \mathrm{~min}$ (three times). Finally, the obtained MPDA particles were re-dispersed in ultrapure water for further use.

\section{The preparation of nanocomposites of MPDA and RL-QN15} The MPDA nanoparticles were completely dispersed in PBS by an ultrasonic device (VCX750, Nanjing Xinchen Biotechnology Co., Ltd, China), then the peptide RL-QN15 were stirred with the dispersed MPDA nanoparticles at $4{ }^{\circ} \mathrm{C}$ for $12 \mathrm{~h}$ on a magnetic stirrer (Thermo Scientific, USA) to form nanocomposites of MPDA and RL-QN15.

\section{Characterization of MPDA}

Field-emission scanning electron microscopy (FE-SEM, SU8020, HITACH, Japan) was used to investigate the surface morphologies and microstructures of MPDA particles. Transmission electron microscopy (TEM) images and mapping results were obtained using a Tecnai G2 F20 field emission transmission electron microscope (FEI, USA). Nitrogen adsorption-desorption isotherms and Brunauer-Emmett-Teller (BET) surface areas were measured with an ASAP 2460 Analyzer (Micromeritics Instruments, USA) at $-196{ }^{\circ} \mathrm{C}$.

\section{Characterization of nanocomposites of MPDA and RL-QN15}

FE-SEM (FE-SEM, SU8220, HITACH, Japan) was used to investigate the surface morphologies and microstructures of nanocomposite of MPDA and RL-QN15. The spectral range of 4000 and $400 \mathrm{~cm}^{-1}$ (mid-infrared) of MPDA and nanocomposites of MPDA + RL-QN15 were analyzed by using a Fourier transform infrared spectroscopy (FT-IR, NICOLET-IS10, Thermo Scientific, USA) in the attenuated total reflection sampling mode. X-ray photoelectron spectroscopy (XPS) measurement was performed on a Thermo Scientific K-Alpha X-ray photoelectron spectrometer (Thermo Scientific, USA) equipped with a monochromatized Al Ka X-ray source (1486.6 eV).

\section{Drug encapsulation efficiency}

RL-QN15 was dissolved in PBS solution and its relative concentration was determined by spectrophotometer (GeneQuant 100, USA). Then $1 \mathrm{mg}$ MPDA was dispersed in RL-QN15 solution and stirred at $4{ }^{\circ} \mathrm{C}$ for $24 \mathrm{~h}$ to form nanocomposites solution, which were then centrifuged (10, $000 \mathrm{rpm}, 5 \mathrm{~min}, 4{ }^{\circ} \mathrm{C}$ ) at different time points, and the supernatant was taken to determine its concentration. The drug encapsulation efficiency of MPDA loaded RL-QN15 was calculated by the equation as follows:

Encapsulation efficiency $(\%)=\left[C_{(0)}-C_{(2,4,6,10,16}\right.$, $\left.{ }_{24)}\right] / C_{(0)} \times 100$. where $C_{(0)}$ represents the initial concentration, and $\mathrm{C}_{(2,4,6,10,16,24)}$ represents the concentration in $2,4,6,10,16,24 \mathrm{~h}$, respectively.

\section{In-vitro drug release studies}

The above successfully encapsulated nanocomposites of MPDA and RL-QN15 were re-suspended in PBS and Dulbecco's modified Eagle's medium (DMEM, BI, Israel). solution respectively and shook at speed of $80 \mathrm{rpm}$ at 37 ${ }^{\circ} \mathrm{C}$. The release of peptide was analyzed by monitoring the content of RL-QN15 in the supernatant after centrifugation $(10,000 \mathrm{rpm}, 2 \mathrm{~min})$ at different time intervals over a period of $24 \mathrm{~h}$. The cumulative released RL-QN15 was calculated by the equation as follows:

Cumulative released of RL-QN15 (\%) $=\mathrm{C}_{(\mathrm{t})} / \mathrm{C}_{(0)}$ $\times 100$. Where $C_{(t)}$ represents the concentration of RL-QN15 in the supernatant at different time points, and $\mathrm{C}_{(0)}$ represents the initial concentration of RL-QN15 in the nanocomposites.

\section{Effects of nanocomposites of MPDA and RL-QN15} on the healing of full-thickness dorsal skin wounds in mice Adult Kunming male mice ( $\mathrm{n}=30,20-24 \mathrm{~g}$ ) form the same generation were purchased from Hunan SJA Laboratory Animal Co., Ltd. The mice were provided with essential food and water and the reverse of 12:12-h light/dark cycle. After five days of acclimatization, two identical round full-thickness cutaneous wounds $(\phi 10$ $\mathrm{mm}$ ) were created on the back of each mice, as previous research [22]. Mice were then randomly assigned into 4 groups, which were either treated with MPDA $(20 \mu \mathrm{L}$, $0.2 \mathrm{mg} / \mathrm{mL})+\mathrm{RL}-\mathrm{QN} 15(20 \mu \mathrm{L}, 1 \mathrm{nM}), \mathrm{RL}-\mathrm{QN} 15(20$ $\mu \mathrm{L}, 1 \mathrm{nM})$, MPDA $(20 \mu \mathrm{L}, 0.2 \mathrm{mg} / \mathrm{mL})$ or PBS $(20 \mu \mathrm{L})$ once daily. Mice were kept in individual ventilated cages (FENGSHI, China) system with laboratory animal room of Kunming Medical University. For observing the wound healing process, the wounds were observed and captured by a digital camera at day $0,2,4,6$ and 8 . Wound condition was documented, with ImageJ software (NIH, USA) employed to estimate wound area (percentage of residual wound area to initial area) and GraphPad Prism software 
used to quantify the rates of wound healing. Mouse skin wound tissues $(n=5)$ were isolated at days 4 and 8 , and analyzed with Hematoxylin \& Eosin (H\&E) and light microscopy.

\section{Effects of nanocomposites of MPDA and RL-QN15 on the healing of burn wounds in mice}

Adult Kunming male mice $(\mathrm{n}=30,20-24$ g) were obtained from Hunan SJA Laboratory Animal Co., Ltd. Mice were provided with essential food and water and maintained under a reverse 12:12-h light/dark cycle. Mice were given burn skin wounds after 7 days of acclimation. As previous research, two burn wounds were formed on the back of each mouse [26]. Briefly, mice were anesthetized with $4 \%$ chloral hydrate (Solarbio, Beijing, China) at $10 \mathrm{~mL} / \mathrm{kg}$. Dorsal hair was shaved by an electric clipper and the skin was sterilized with $75 \%$ alcohol. A special metallic device containing a $10-\mathrm{mm}$ diameter disc was used for inducing the experimental wound. The device was heated in boiling water and applied on the shaved dorsal area for $15 \mathrm{~s}$. Mice were then randomly assigned into 4 groups, which were either treated with MPDA $(20 \mu \mathrm{L}, 0.2 \mathrm{mg} / \mathrm{mL})+\mathrm{RL}-\mathrm{QN} 15(20 \mu \mathrm{L}, 1 \mathrm{nM})$, RL-QN15 $(20 \mu \mathrm{L}, 1 \mathrm{nM})$, MPDA $(20 \mu \mathrm{L}, 0.2 \mathrm{mg} / \mathrm{mL})$ or PBS $(20 \mu \mathrm{L})$ twice daily. For observing the wound healing process, wounds were observed and captured by a digital camera at day $1,4,8$, and 12 . Wound areas (percentage of residual wound area to original wound area) were estimated from the photographs using ImageJ software (NIH, USA), in which the edges of the wounds were traced and the area of the pixels was calculated. Mouse skin wound tissues $(n=5)$ were isolated at days 8 and 12 , and analyzed with $\mathrm{H}$ and $\mathrm{E}$ staining and light microscopy.

\section{Effects of nanocomposites of MPDA and RL-QN15} on the healing of acute full-thickness dorsal skin in swine Bama swine $(\mathrm{n}=2,12-14 \mathrm{~kg})$ were obtained from the Experimental Animal Center of Kunming Medical University. Swine were provided with essential food and water and the reverse of 12:12-h light/dark cycle. The swine were made full-thickness skin wound models after 7 days' acclimation. Before the operation, animals were fasted for $12 \mathrm{~h}$, but given access to water. Chlorpromazine $(0.15 \mathrm{~mL})$ was intramuscularly injected for sedation. After $5 \mathrm{~min}$, the pigs received a gluteal intramuscular injection of $3 \%$ pentobarbital sodium $(1 \mathrm{~mL} / \mathrm{kg})$. Hair was then shaved, and skin was washed with saline and sterilized with $75 \%$ alcohol. Using a permanent marker and a $30 \times 30 \mathrm{~mm}$ template, wounds were drawn $30 \mathrm{~mm}$ apart on the dorsal skin to allow for adequate healing. A sterile No. 22 scalpel blade was used to excise the marked areas down to the fat layer. Finally, 12 wounds of the same size were formed on the back of each swine. The wounds were randomly assigned into 4 groups, which were topically treated with PBS, PDA $(0.2 \mathrm{mg} / \mathrm{mL})$, RL-QN15 $(250 \mathrm{nM})$, or nanocomposites of MPDA $(0.2 \mathrm{mg} / \mathrm{mL})$ and RL-QN15 (250 nM) once a day. Wound condition was documented, with ImageJ employed to estimate wound area (percentage of residual wound area to initial area) and GraphPad Prism used to quantify wound healing. The tissues of wounds on the post-operative 28 day were further analyzed histologically by H\&E staining.

\section{Histological analysis}

All tissue samples isolated from animal were fixed in $4 \%$ paraformaldehyde for $24 \mathrm{~h}$, and then dehydrated and hyalinized as per previous study [27]. For histological analysis, the center of the repair site was sliced into $5-\mu \mathrm{m}$ sections followed by H\&E and Masson trichrome staining. All slices were imaged by a Primovert microscope (Zeiss, Germany) and used to observe epidermal regeneration and granulation formation. For the evaluation of neo-epidermal thickness and granular thickness in acute skin wounds, five values were randomly measured in a field and mean values were calculated using Image software (NIH, USA).

\section{Result and discussion}

\section{Preparation and characterization of MPDA}

Owing to their characteristics of surface functionalization, targeting, good degradability and biocompatibility, nanoparticles have been widely used in modern biomedicine as interventions of anti-tumor, antioxidation, drug delivery and tissue regeneration, et al. Recently, the application of nanoparticles in the field of wound healing has gradually attracted extensive attention, for example, the nanofibrous matrix based on biomimetic elastomeric peptide has been developed to overcome the multidrugresistant bacterial and promote the skin regeneration [28], in addition, grape seed-inspired smart hydrogel scaffolds has also been reported to significantly accelerate the healing of wound [29]. MPDA nanoparticles shows unique characteristics featured by simple preparation protocol, strong adhesive properties, easy and straightforward functionalization, and biocompatibility, and thus has been widely used in the fields of biomedicine, sensing, catalysis, environment and energy [30]. However, for such an important nanoparticle, there are few reports on its application in accelerating the regeneration of skin wounds.

In the current research, MPDA nanoparticles were synthesized according to previously reported procedures [25]. As described in the "Experimental section", MPDA nanoparticles were formed in an aqueous solution containing triblock copolymer Pluronic F-127 and 1, 3, 5-trimethylbenzene (TMB) as organic templates. Dopamine 

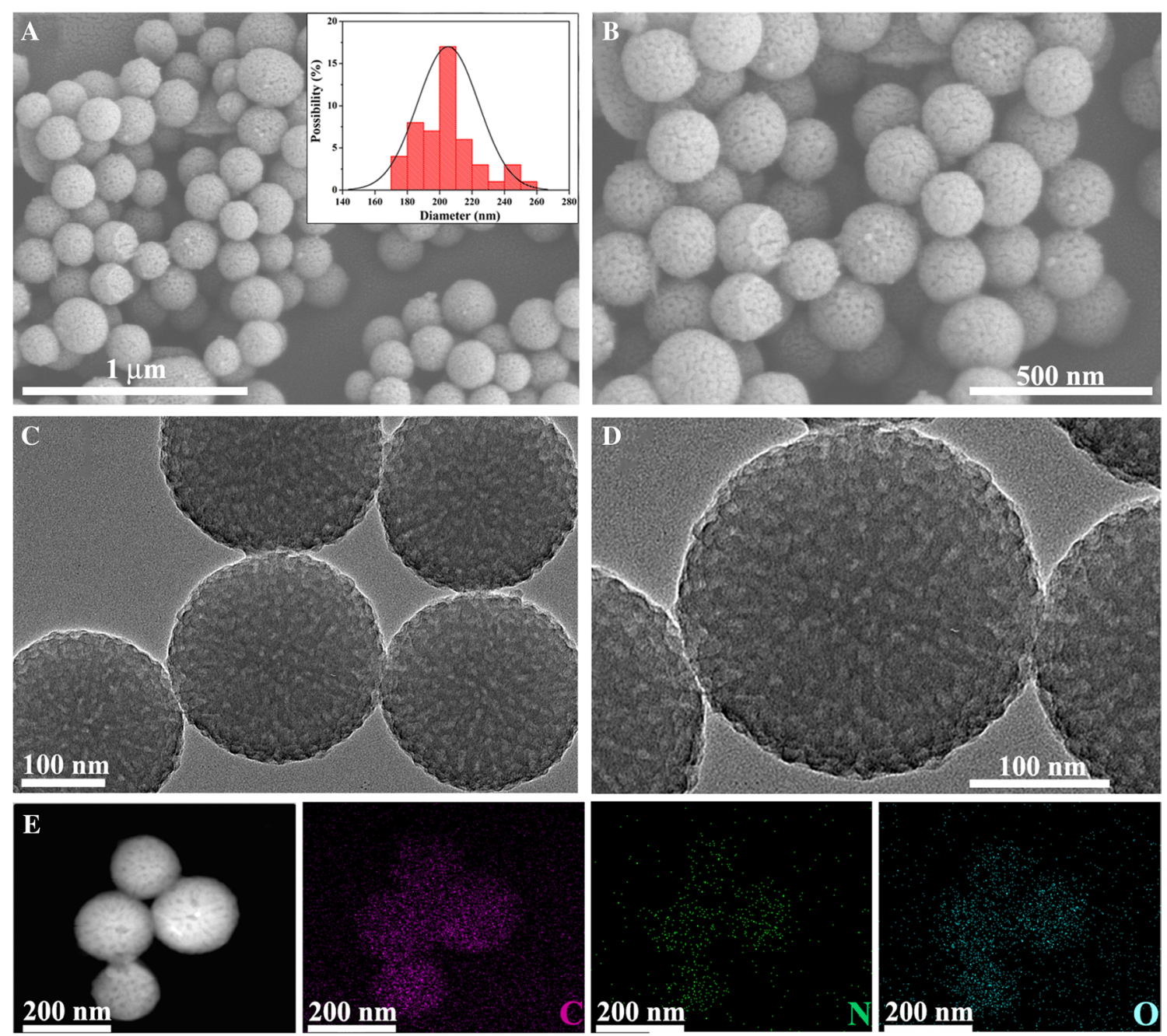

F

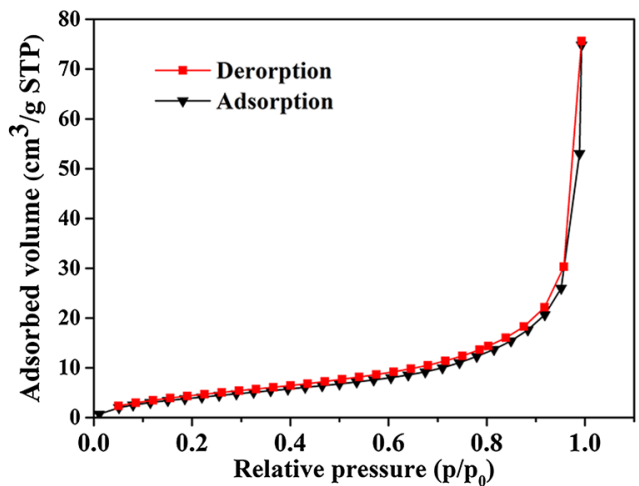

G

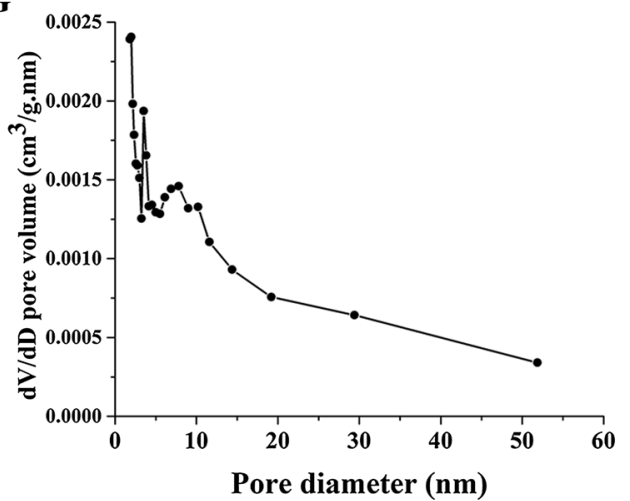

Fig. 1 The characterization of MPDA. A, B FE-SEM images and (C, D) TEM images of MPDA particles. High magnification SEM images in B and TEM images in $\mathbf{D}$ showed typical MPDA particles. Inset in $\mathbf{A}$ showed corresponding size distribution histograms and theoretical fitting of MPDA particles. E Dark field TEM and energy-dispersive X-ray spectroscopy-based elemental mapping analysis of MPDA particles. Scale bars were indicated by lines in the figures 
self-polymerized into PDA particles through an ammonia-catalyzed approach, and the resultant PDA particles were assembled on Pluronic F-127-stabilized TMB droplets via $\pi-\pi$ stacking interactions. The PDA particles were finally obtained as the organic templates were removed. Both FE-SEM (Fig. 1A, B) and TEM images (Fig. 1C, D) confirmed that the resultant PDA particles showed well-defined spherical morphologies and mesoporous structures. Their average diameter and polydispersity were $205 \mathrm{~nm}$ and 3.9\%, respectively (see inset in Fig. 1A). Close observation of the MPDA nanoparticles at higher magnification (Fig. 1B, D) revealed that the cylindrical open channels were exposed on the MPDA nanoparticle surfaces, in which some mesopores had diameters of $10 \mathrm{~nm}$. The elemental mapping patterns revealed a uniform distribution of $\mathrm{C}, \mathrm{N}$, and $\mathrm{O}$ elements (Fig. 1E), further verifying the formation of MPDA nanoparticles. As shown in Fig. 1F, G, the nitrogen adsorption-desorption isotherms of MPDA demonstrated hysteresis loops of type IV, which is characteristic of mesoporous materials. The results revealed that the prepared MPDA nanoparticles had a surface area of $21.5475 \mathrm{~m}^{2} / \mathrm{g}$, pore volume of $0.119326 \mathrm{~cm}^{3} / \mathrm{g}$, and pore diameter of $21.3857 \mathrm{~nm}$ (Additional file 1: Table S1). Thus, due to their mesoporous structure, large surface area, and nano-sized spherical morphology, MPDA particles exhibit great potential as versatile platforms for drug delivery, diagnosis, and therapy [31].

\section{Characterization of nanocomposites of MPDA + RL-QN15}

As a molecular tool, peptides have not only contributed substantially to clarify physiological processes in humans but also led to the discovery and development of novel therapeutics, hence it is widely recognized that peptide molecules have made indelible contributions to both basic scientific research and development of new drugs that are represented by insulin, exenatide, hirudin, captopril, et al. [32]. Amphibian skin secretions are rich in a variety of bioactive peptides, such as antimicrobial peptides, antioxidant peptides, bradykinins, neuromodulating peptides, and neurotoxins, et al. and thus have been considered as a treasure trove of the natural bioactive peptide [16, 33, 34]. In recent years, peptides with obvious potency of accelerating the healing of skin wounds have aroused significant attention and several prohealing peptides have been identified, including RL-QN15, cathelicidin-OA1 and cathelicidin-NV, Ot-WHP, tylotoin, OM-LV20, et al. [22, 35-39]. Our previous research have revealed that RL-QN15 contains an amino acid sequence of QNSYADLWCQFHYMC and an intramolecular disulfide bond located between $C^{9}$ and $C^{15}$, when at a relative low concentration of $\mathrm{nM}$ scale, more importantly, RL-QN15 significantly promote the healing of full-thickness injured wounds and diabetic skin chronic wounds in mice, and thus is considered as a promising prohealing agent [23]. However, we still hoped that the ability of RL-QN15 to promote repair activity can be improved by some ways, so as to provide new strategies for the development of novel therapeutics for the treatment of wound healing which are still a harsh clinical challenge. One of the available methods was to use the nanoparticles to enhance the prohealing potency of RL-QN15.

In the current research, considering the characteristics of MPDA adhesion to each other, we first dispersed the prepared MPDA in PBS, as shown in Fig. 2A, the PBS solution of RL-QN15 is colorless, MPDA and MPDA + RL-QN15 nanocomposites are brown. Here, RL-QN15 with a molecular weight of only $1906 \mathrm{Da}$ is completely soluble in water and PBS. Then, FT-IR analysis, which is useful in qualitative analysis as no two bioactive compounds have the same FT-IR spectra [40], was carried out to verify whether RL-QN15 was successfully loaded onto the MPDA shell. Figure $2 \mathrm{~B}$ showed the characteristic peaks of MPDA and MPDA+RL-QN15 nanocomposites. The broad peak at $3440 \mathrm{~cm}^{-1}$ in the PDA spectra was the characteristic adsorption of amine $\mathrm{N}-\mathrm{H}$ and phenolic $\mathrm{O}-\mathrm{H}$ stretching vibrations, and the band centered at $1630 \mathrm{~cm}^{-1}$ was assigned to the formation of an indole-related structure after the oxidation and self-polymerization of dopamine [41, 42]. Compared with MPDA, the wavenumber of the MPDA + RL-QN15 nanocomposites was lower at these two sites, which was attributed to the intermolecular hydrogen bond between MPDA and RL-QN15. In addition, there was a significant difference in the spectral fingerprint $\left(1800-500 \mathrm{~cm}^{-1}\right)$ between MPDA and MPDA+RL-QN15 nanocomposite. The characteristic absorption peaks of MPDA could be observed in the spectrum at the dotted frames, while the characteristic absorption peaks of MPDA/RL-QN15 nanocomposites were obviously weakened or even disappeared in the two places. These results suggested that RL-QN15 was present in MPDA nanoparticles. In addition, results of SEM also provided evidence of the successful formation of MPDA+RL-QN15 nanocomposite. Compared with the MPDA shell (Fig. 1A, B), the surface of the MPDA+RL-QN15 nanocomposites was rough and contained small particles (Fig. 2C, D). The diameter of MPDA and nanoparticles of MPDA + RL-QN15 shared similar diameter of about $200 \mathrm{~nm}$. The X-ray photoelectron spectroscopy (XPS) experiments provided additional evidence for the successful loading of peptides onto MPDA nanoparticles. Our results showed that the characteristic binding energy peaks at 283,398 , and $530 \mathrm{eV}$ are attributed to the $\mathrm{C}, \mathrm{N}, \mathrm{O}$ elements present in MPDA and MPDA + RL-QN15, and the nanocomposites 



Fig. 2 Characterization of nanocomposites of MPDA + RL-QN15. A The representative images of MPDA dispersed in PBS, RL-QN15 dissolved in PBS and nanocomposites of MPDA + RL-QN15 in PBS. B The FT-IR spectra of MPDA and nanocomposites of MPDA+RL-QN15. C, D SEM images of nanocomposites of MPDA + RL-QN15 at magnification of $\times 40,000$ and 80,000 , respectively. Scale bars were indicated by lines in the figures

have more binding energy peaks, with the peak at $161 \mathrm{eV}$ due to the $\mathrm{S}$ element present in the Met and Cys of the peptide. RL-QN15 was successfully loaded into the mesoporous channel inside the MPDA NPs by sufficient stirring with the MPDA particles and hydrogen bonding of the catechol, amine, and imine groups on the nanoparticle surface.
In summary, we successfully prepared nanocomposite of MPDA + RL-QN15, and then we focused on the loading efficiency and release profile of the composites.

\section{In-vitro drug release and encapsulation efficiency of RL-QN15 loaded MPDA nanocomposites}

The loading and releasing of RL-QN15 is based on the porous structure of MPDA microspheres. Here, based 

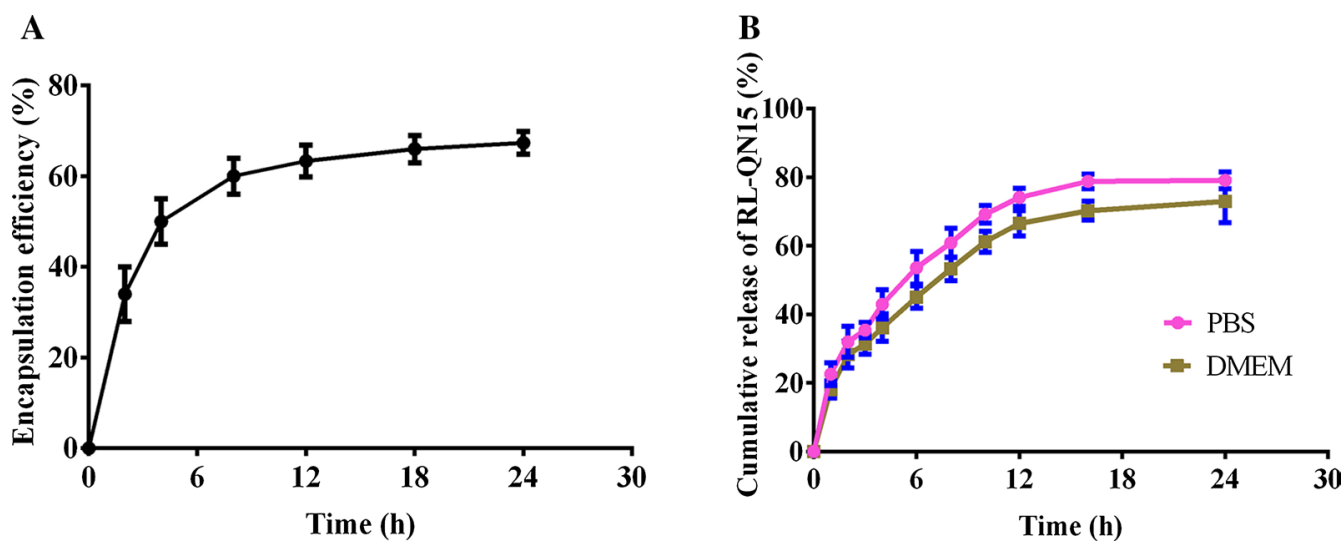

Fig. 3 Loading efficiency and RL-QN15 release profile of MPDA/RL-QN15 nanocomposites. A The encapsulation efficiency of nanocomposites of MPDA and RL-QN15 at different time. B Release profiles of RL-QN15 from nanocomposites of MPDA and RL-QN15 in PBS solution and cell culture medium

on the specific absorption peak of the peptide bond at 220nm, we made a standard curve of concentration vs. absorbance. As shown in Fig. 3A, the encapsulation rate of MPDA + RL-QN15 nanocomposites slowed down significantly after $6 \mathrm{~h}$, and the maximum encapsulation was $67.31 \%$. In addition, the slow release of RL-QN15 from MPDA microspheres has also been studied. As shown in Fig. 3B, RL-QN15 was continuously released from the microsphere during the period of $0-16 \mathrm{~h}$, and the release rate was $79.12 \%$ at $24 \mathrm{~h}$.

The MPDA microsphere nanoparticles have the characteristics of efficient loading and slow release of RL-QN15 and in the following experimental procedures, the main focus was to verify if the load of MPDA significantly enhanced the prohealing potency of RL-QN15.

\section{The load of MPDA significantly enhanced the prohealing potency of RL-QN15 on full-thickness injured wounds in mice}

The full-thickness skin wound model of mice is adopted to preliminarily evaluate whether the load of MPDA could significantly increase the activity of RL-QN15. PBS, PDA, RL-QN15, nanocomposite of MPDA + RL-QN15 was administered topically twice a day to the wounds constructed on the dorsal skin of mice. As shown in Fig. 4A, B, compared with PBS, MPDA $(0.2 \mathrm{mg} / \mathrm{mL})$ could not whereas RL-QN15 (1 nM) could significantly promote the skin wound healing. On the eighth postoperative, RL-QN15 increased the healing effect by nearly $20 \%\left({ }^{* *} \mathrm{P}<0.01\right)$ compared with the control group, showing excellent potential to accelerate wound healing, which was consistent with the data in our previous report [23]. It was worth pointing out that the healing ability of MPDA loaded with lower concentration of RL-QN15 (1 nM) was similar to that of RL-QN15 (50 nM) alone. In other words, MPDA nanoparticles as the carrier of peptides can significantly enhanced the prohealing potency of RL-QN15 by nearly 50 times. Specifically, on the postoperative days $2,4,6$, and 8 , the wound healing rates in the RL-QN15 was $58.35 \pm 5.08 \%, 67.81 \pm 5.14 \%$, $76.39 \pm 5.56 \%, 84.3 \pm 2.38 \%$, respectively, however, by means of the load of MPDA, healing rate in nanocomposites of MPDA + RLQN15 increased to $69.69 \pm 5.1 \%$, $80.24 \pm 4.36 \%, \quad 87.07 \pm 5.85 \%, \quad 98.48 \pm 2.46 \%$, respectively, which showed increases of $19.43 \%, 18.33 \%, 13.98 \%$, $17.14 \%$, respectively. In addition, the repair-promoting activity of nanocomposites of MPDA+RLQN15 was time-dependent (Fig. 4B).

\footnotetext{
(See figure on next page.)

Fig. 4 Topical application of nanocomposites of MPDA + RL-QN15 accelerated healing of full-thickness skin wounds in mice. A Representative images of skin wounds on days 0, 2, 4, 6, and 8 post-injury in PBS, MPDA, RL-QN15, and nanocomposites of MPDA and RL-QN15-treated groups. MPDA $(0.2 \mathrm{mg} / \mathrm{mL}), \mathrm{RL}-\mathrm{QN} 15(1 \mathrm{nM})$, and MPDA $(0.2 \mathrm{mg} / \mathrm{mL})+$ RL-QN15 $(1 \mathrm{nM})$ were applied topically twice a day to skin wounds. B Quantitative curves of pro-healing activities of PBS, MPDA, RL-QN15, and MPDA + RL-QN15, respectively. C Histopathological examination of PBS, MPDA, RL-QN15 and RL-QN15 + PDA-treated full-thickness excisional wounds stained with H\&E. NE, neo-epidermal; GT, granulation tissue; ES, eschar; M, muscle; Scale bar: $500 \mu \mathrm{m}$. Yellow line indicates neoepithelium. D, E The quantitative analysis of neo-epidermal thickness on days 4 and 8 post-operative. $\mathbf{F}, \mathbf{G}$ The quantitative analysis of granulation thickness on day 4 and 8 post-operation, respectively. Dates were presented as mean \pm S.D. from 9 mice $(n=9) .{ }^{*} P<0.05,{ }^{* *} P<0.01$, and ${ }^{* * *} P<0.0001$ indicated statistical differences between two groups
} 

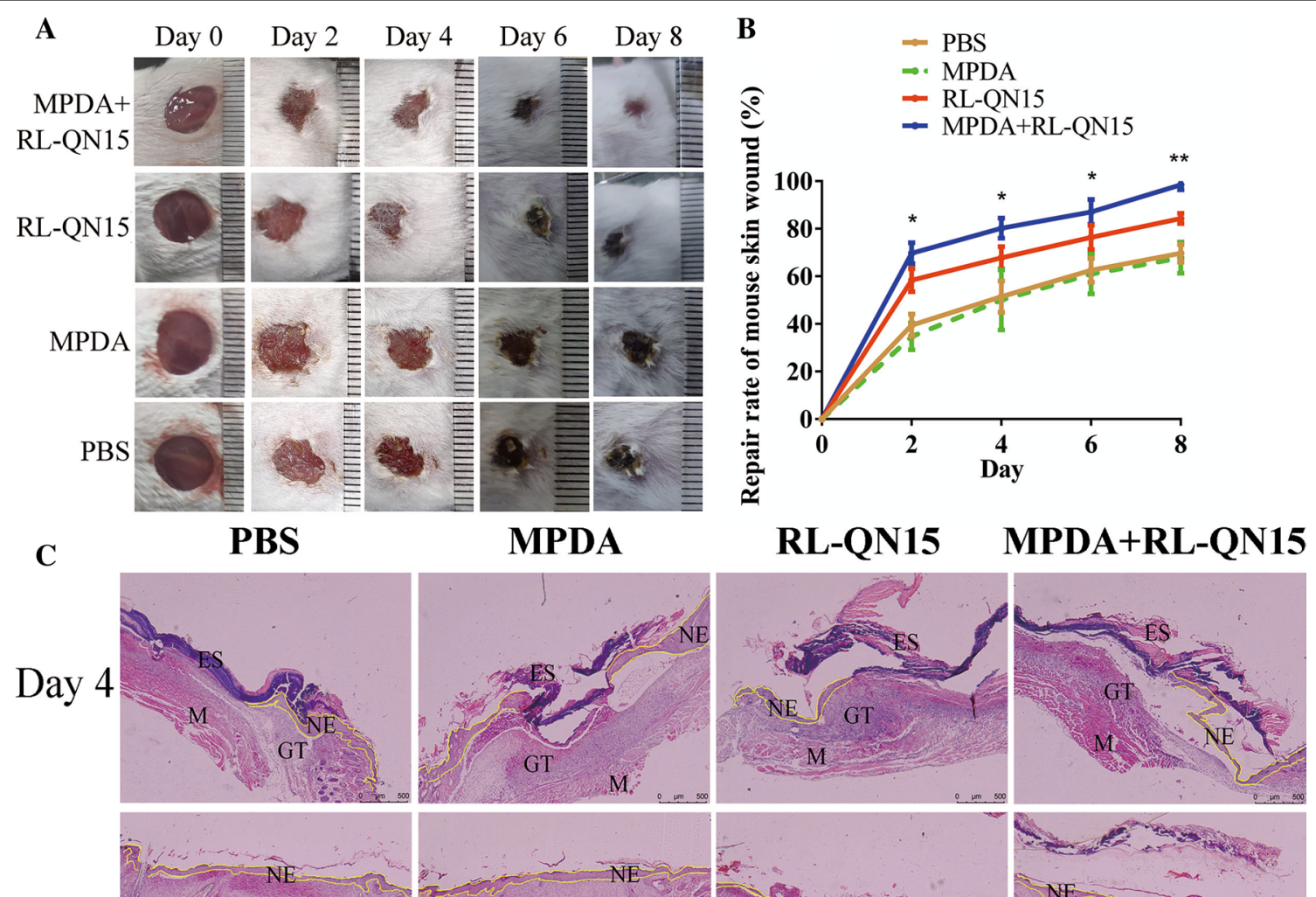

Day 8


$$
\text { D }
$$
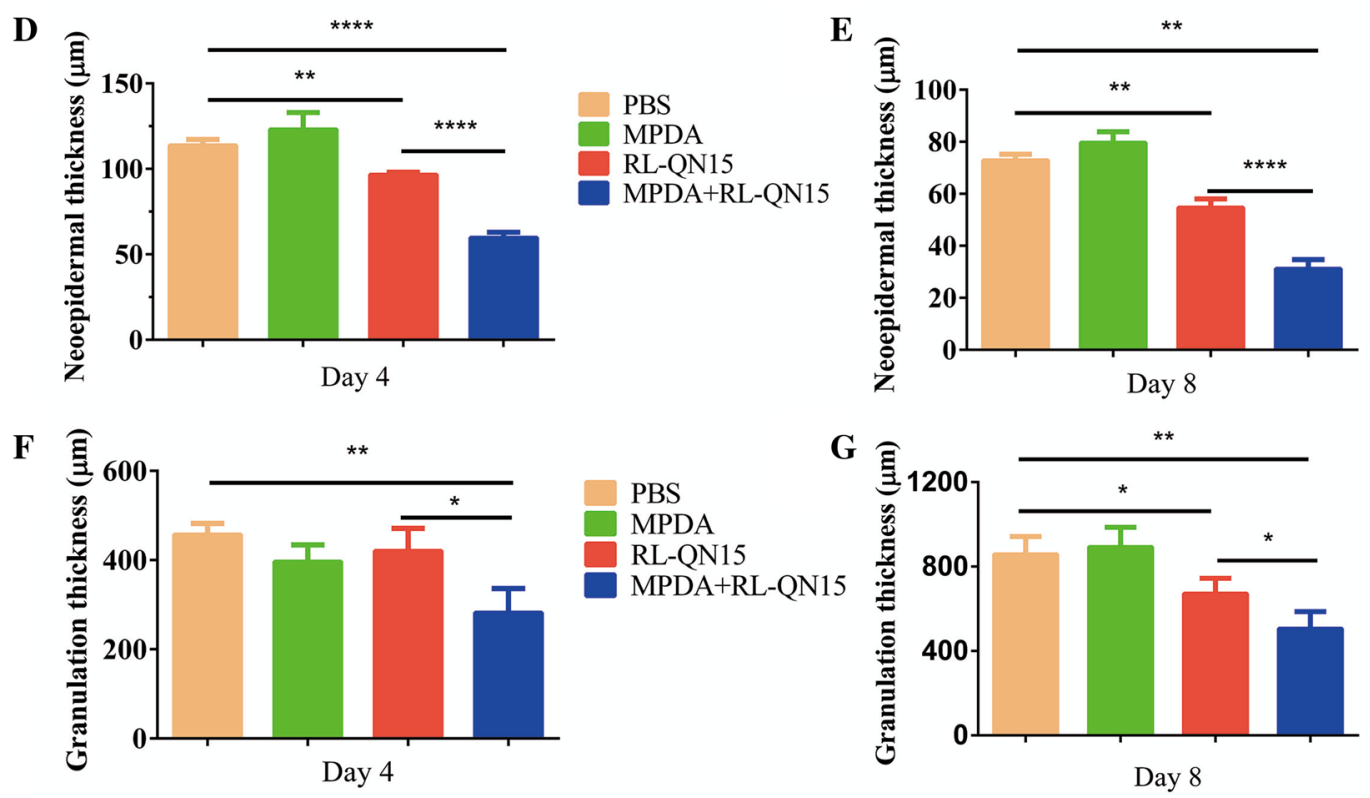

Fig. 4 (See legend on previous page.) 
Mice were sacrificed for histological analysis at postoperative days 4 and 8 , and the effects of MPDA + RL-QN15 on wound healing in vivo were further investigated. Chronic or non-healing wounds and excessive scarring have consistently been pressing problems in wound healing. On the one hand, once a wound is formed, a great number of fibroblasts and keratinocytes keep proliferating and migrating, which contributes to the generation of granulation tissue and neoepidermal epidermis on the wound surface. Then, on the other hand, excessive proliferation and migration lead to over deposition of extracellular matrix, which is a major cause of scar formation. At the histological level, pathological scarring is characterized by having thick granulation tissue and epidermis. Therefore, the assessment of epidermal re-epithelialization, neoepidermis thickness, and granulation thickness is crucial for wound healing and its healing quality. Here, the H\&E images of each group of wounds were quantified by ImageJ software. As shown in Fig. $4 \mathrm{C}$, D, on postoperative day 4, the neoepidermis thickness of mice in PBS, PDA, RL-QN15 groups were $113.29 \pm 4.36 \mu \mathrm{m}, 139.84 \pm 27.78 \mu \mathrm{m}, 96.68 \pm 1.59 \mu \mathrm{m}$, respectively, while the neoepidermis thickness of mice in MPDA + RL-QN15 group was only $59.85 \pm 3.25 \mu \mathrm{m}$ (Fig. 4D). On the postoperative day 8 , there was no significant difference in the thickness of epidermis between PBS and MPDA groups $(72.89 \pm 2.35 \mathrm{~m}$ vs. $83.74 \pm 2.52 \mu \mathrm{m})$. The thickness of epidermis in RL-QN15 was about $54.70 \pm 3.63 \mu \mathrm{m}$, which was significantly lower than that in PBS and PDA groups, but still higher than that in MPDA + RL-QN15 group (31.24 $\pm 3.87 \mu \mathrm{m})$. We also evaluated the granulation tissue thickness, as shown in Fig. 4F, G, on postoperative day 4, there was almost no difference in granulation tissue thickness between the PBS, PDA and RL-QN15 groups, and MPDA + RL-QN15 was lowest among the four groups. On postoperative day 8 , there was no significant in granulation thickness between PBS and PDA groups $(858.53 \pm 96.13 \mu \mathrm{m}$ vs. $863.71 \pm 89.14 \mu \mathrm{m})$. Granulation thickness of RL-QN15 was $672.76 \pm 74.43 \mu \mathrm{m}$, which was significantly lower than that of PBS and PDA groups, but still higher than that of MPDA + RL-QN15 group $(506.60 \pm 83.62 \mu \mathrm{m})$. In a word, after topical administration of nanocomposites of MPDA + RL-QN15, the regeneration and reconstruction of the epidermis and granulation tissue were significantly enhanced.

In previous studies, there are few reports on the combination of nanomaterials and peptides to promote wound healing. One of available references is that KR-12 peptide combined to the fibrous eggshell membrane to promote angiogenesis and hence accelerate skin re-epithelialization [43]. The report on the binding of MPDA particles to peptides as prohealing therapeutics remains unavailable. Our results showed that, by the load by MPDA, the prohealing potency of RL-QN15 against the full-thickness injured wounds in mice were increased by an average of $17.14 \%$. Hence, for the first time, we provided evidence to indicate that MPDA nanoparticles could be used as a peptide-carrier to achieve better pro-healing potency.

\section{The prohealing potency of RL-QN15 against burn skin wounds was significantly enhanced by the load of MPDA}

Burn is a common trauma in daily life and the clinically available means to treat burn is the application of skin grafting and wound dressing. However, slow wound healing, infection, pain, and hypertrophic scarring continue to remain a major challenge in burn research and management [44]. In our previous research, we demonstrated that RL-QN15 could promote chronic skin wound healing in diabetic mice, but it is unknown whether RL-QN15 could promote burn wound healing. In this study, we successfully established the mice model of deep-II degree burn on the dorsal skins. The macroscopic morphology of the experimentally induced burns was presented in Fig. 5A. After inducing the burns on experimental animals, the local area was characterized by a white eschar, the surface skin layers (epidermis and dermis) were damaged. In the first four days, the wound turned brown due to extravasation of injured cells and an increase in the level of inflammation. Eight days after the burn, the skin formed a scab and gradually fell off. PBS, MPDA, RL-QN15, nanocomposite of MPDA+RL-QN15 was administered twice a day to the burns of mice. As shown in Fig. 5B, MPDA itself could not promote skin wound healing in mice compared with PBS, while RL-QN15 (1 nM) could significantly promote wound healing. On day 12 post-operation, the wound healing rate in the

(See figure on next page.)

Fig. 5 The ability of RL-QN15 against burn wound was obviously improved by the load of MPDA. A Representative images of scald wounds on days 0, 4, 8, and 12 post-injury in PBS, MPDA, RL-QN15, and MPDA+RL-QN15 groups. MPDA (0.2 mg/mL), RL-QN15 (1 nM), and nanocomposites of MPDA $(0.2 \mathrm{mg} / \mathrm{mL})+\mathrm{RL}-\mathrm{QN} 15$ (1 nM) were applied topically twice a day to skin wounds. B Quantitative curves of pro-healing activities of PBS, MPDA, RL-QN15, and nanocomposites of MPDA + RL-QN15. C Representative images of burns sections stained with H\&E indicating histomorphological changes in skin wounds on days 8 and 12 post-injury in PBS, MPDA (0.2 mg/mL), RL-QN15 (1 nM), and nanocomposites of MPDA (0.2 mg/ mL) + RL-QN15 (1 nM) groups. NE, neo-epidermal; GT, granulation tissue; ES, eschar; M, muscle. D, E Histological re-epithelialization of epidermis on days 8 and 12 post-injury. Data were presented as mean \pm S.D. from 9 mice $(n=9)$. ${ }^{*} P<0.05,{ }^{* *} P<0.01$, and ${ }^{* * * *} P<0.0001$ indicated statistically differences between two groups 


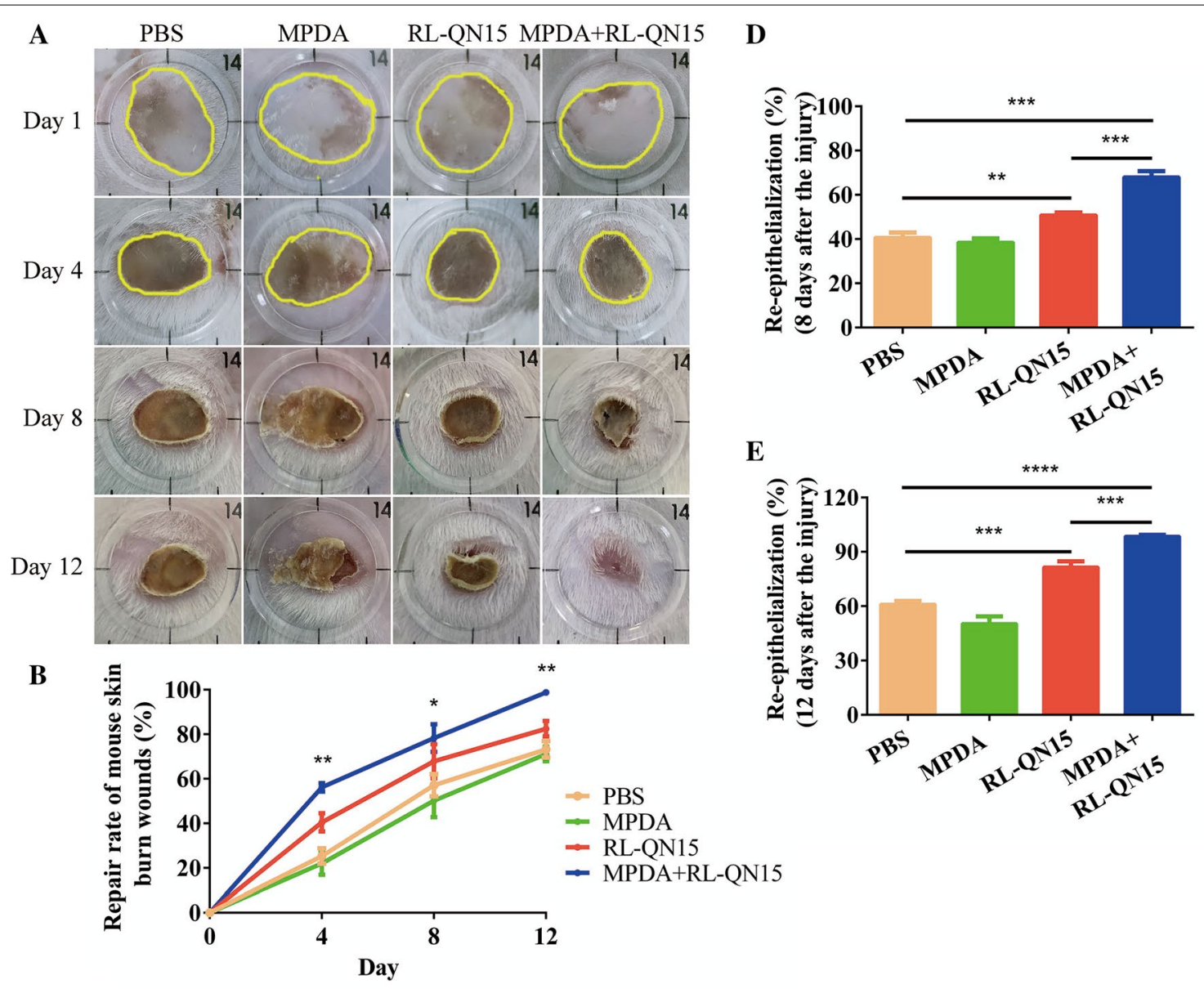

C

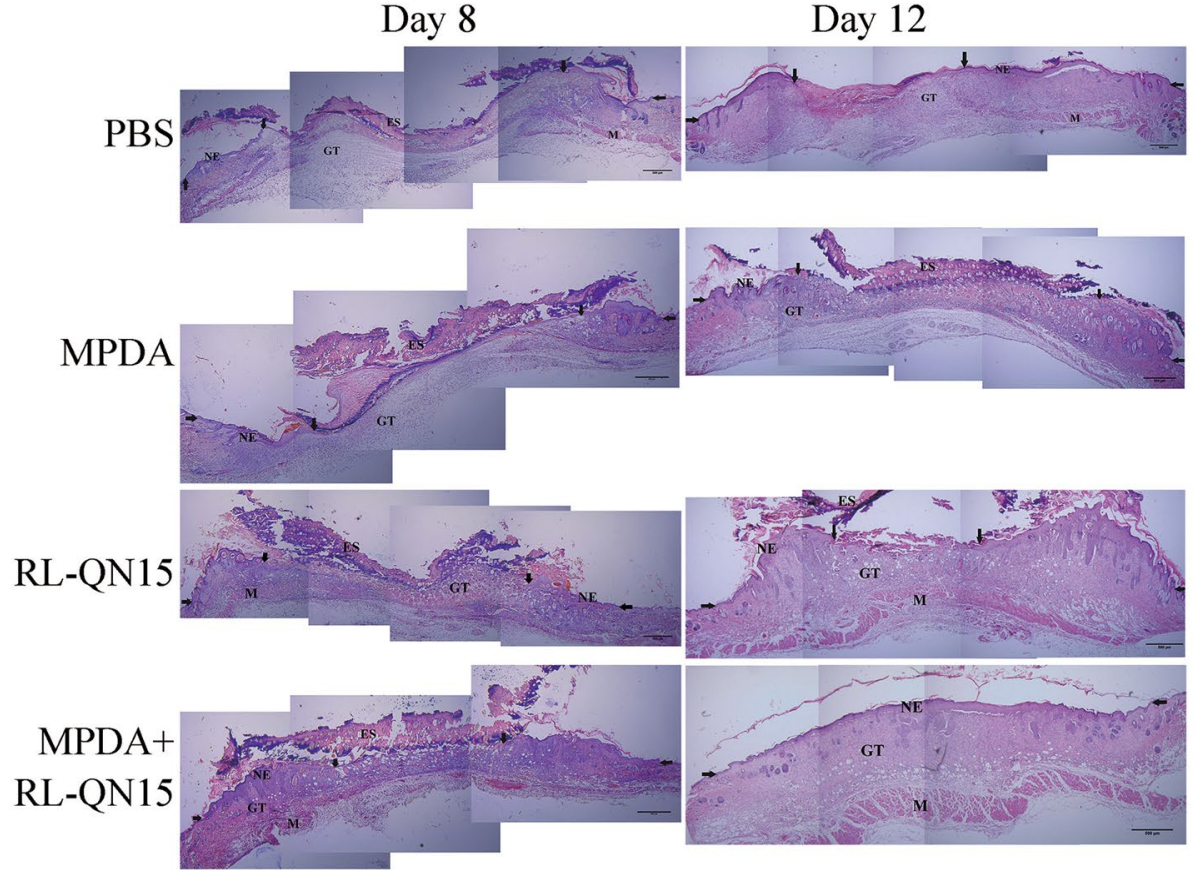

Fig. 5 (See legend on previous page.) 
PBS and MPDA groups was only $73.23 \pm 4.25 \%$ and $71.16 \pm 3.73 \%$, respectively, while the wound healing rate in the RL-QN15 group was $82.47 \pm 3.53 \%$, which showed that RL-QN15 has the potential to promote the healing of burn wounds in mice. What is worth mentioning is that the prohealing potency of RL-QN15 against chronic skin wounds was significantly enhanced by the load of MPDA. On post-operative day 12, the healing rate of MPDA + RL-QN15 was $98.80 \pm 0.80 \%$, which was $19.80 \%$ higher than that of RL-QN15 (**: $\mathrm{P}<0.01)$.

In a parallel experiment, mice were sacrificed for histological analysis at postoperative days 8 , and 12 , and the effects of nanocomposites of MPDA+RL-QN15 on wound healing in vivo were further investigated. Histological analysis indicated that mice topically treated with nanocomposites of MPDA+RL-QN15 displayed prominently accelerating regeneration of neo-epidermis (neoepithelial tongue) in the wound compared with the PBS, MPDA or RL-QN15 group (Fig. 5C). One of the major systemic damage responses after burn injuries is caused by proinflammatory cytokines released by inflammatory and vascular endothelial cells [45]. During the healing process, the burn wound showed more inflammatory infiltration than the full-thickness wound (Figs. 4C vs. $5 \mathrm{C})$. On the post-injury day 8 , all groups were still in the stage of inflammation, while MPDA+RL-QN15treated group showed the best re-epithelialization and best-formed granulation tissue amongst four groups. On the post-injury day 12 , epidermal regeneration and reconstruction of the dermis were complete in the MPDA + RL-QN15 group, which were similar to that in normal mice. Histological evaluation of mice skin tissue sections stained with H\&E was also carried out. As illustrated in Fig. 5D, E, during the healing process, the level of re-epithelialization in MPDA+RL-QN15 group was always higher than that in other groups. On postoperative day 12 , neo-epithelial tongue was completely covered the whole wounded area in the MPDA+RL-QN15treated mice (re-epithelialization $98.80 \pm 1.15 \%$ ). In contrast, only little partial neo-epithelial tongue was found in the MPDA (re-epithelialization of $50.33 \pm 4.64 \%$ ) and most partial neo-epithelial tongue in the RL-QN15 (re-epithelialization of $82.47 \pm 3.67 \%$ ). In conclusion, histological analysis revealed that mice treated with MPDA+RL-QN15 showed better granulation tissue contraction, and high re-epithelialization.

This is the first time to prove that RL-QN15 showed pro-healing activity on burns in the dorsal skins of mice. Moreover, the load of MPDA significantly enhanced the prohealing potency of RL-QN15. Although RL-QN15 could promote the healing of full-thickness, burns and diabetic wounds in mice, the difference in skin structure between mice and humans made it impossible to provide scientific means for clinical treatment of wound. Considering the clinical application, we next explored the healing activity of RL-QN15 in porcine full-thickness injury.

\section{The effect of RL-QN15 against full-thickness dorsal skin wounds in swine was significantly accelerated by the load of MPDA}

Due to the panniculus carnosus, healing in these small animals is largely achieved through wound contraction, as opposed to re-epithelialization in humans [46, 47]. Furthermore, the murine epidermis is only $50 \mu \mathrm{m}$ thick, so it is technically difficult to create partial-thickness wounds [48]. Porcine models have emerged as promising models to study wound healing. An advantage of using swine is that they are anatomically and physiologically similar to humans [49], and have been used to study many other diseases [50-52]. Like humans, they have a relatively thick epi-dermis, distinct rete pegs, dermal papillae, and dense elastic fibers in the dermis [53, 54]. Swine also have sparse hair rather than fur, although the hair is coarser than human hair. Similarities between swine and human skin also make swine an appropriate choice for the construction of cutaneous wound healing animal models.

A full-thickness dermal skin wound model was established in swine to assess whether the load of MPDA could significantly increase the prohealing activity of RL-QN15. The full-thickness wounds of the dorsal skins were treated with PBS, PDA, RL-QN15, nanocomposite of MPDA+RL-QN15 twice a day. As shown in Fig. 6A, $\mathrm{B}$, compared with PBS, MPDA could hardly promote wound healing of swine skin, while RL-QN15 significantly promoted wound healing. For example, on day 28 post-operation, the wound healing rate in PBS and MPDA groups was only $49.01 \pm 4.48 \%$ and $54.85 \pm 4.11 \%$ $(\mathrm{n}=6)$ respectively, while in RL-QN15 group, the wound healing rate was $65.02 \pm 4.70 \%(n=6)$. In particular, the effect of RL-QN15 against full-thickness dorsal skin wounds in swine was significantly accelerated by the load of MPDA. For instance, at day 7 and 14, there was almost no difference between the two groups, however, at postoperative days 21 and 28, the wound healing rates in the nanocomposite of MPDA + RL-QN15 group was $57.67 \pm 2.98 \%$ and $79.35 \pm 3.30 \%(\mathrm{n}=6)$, respectively, which showed increases of $15.43 \%(" \mathrm{P}<0.05), 21.86 \%$ $\left({ }^{* * *} \mathrm{P}<0.01\right)$ compared with RL-QN15 group.

Histological analysis was also carried out on skin sections (stained using H\&E and Masson's trichrome) from swine on day 28 post-operation. New blood vessels and hair follicles appeared in the group treated with MPDA + RL-QN15 rather than RL-QN15 groups (Fig. 6C). In addition, the thickness of new epidermis 


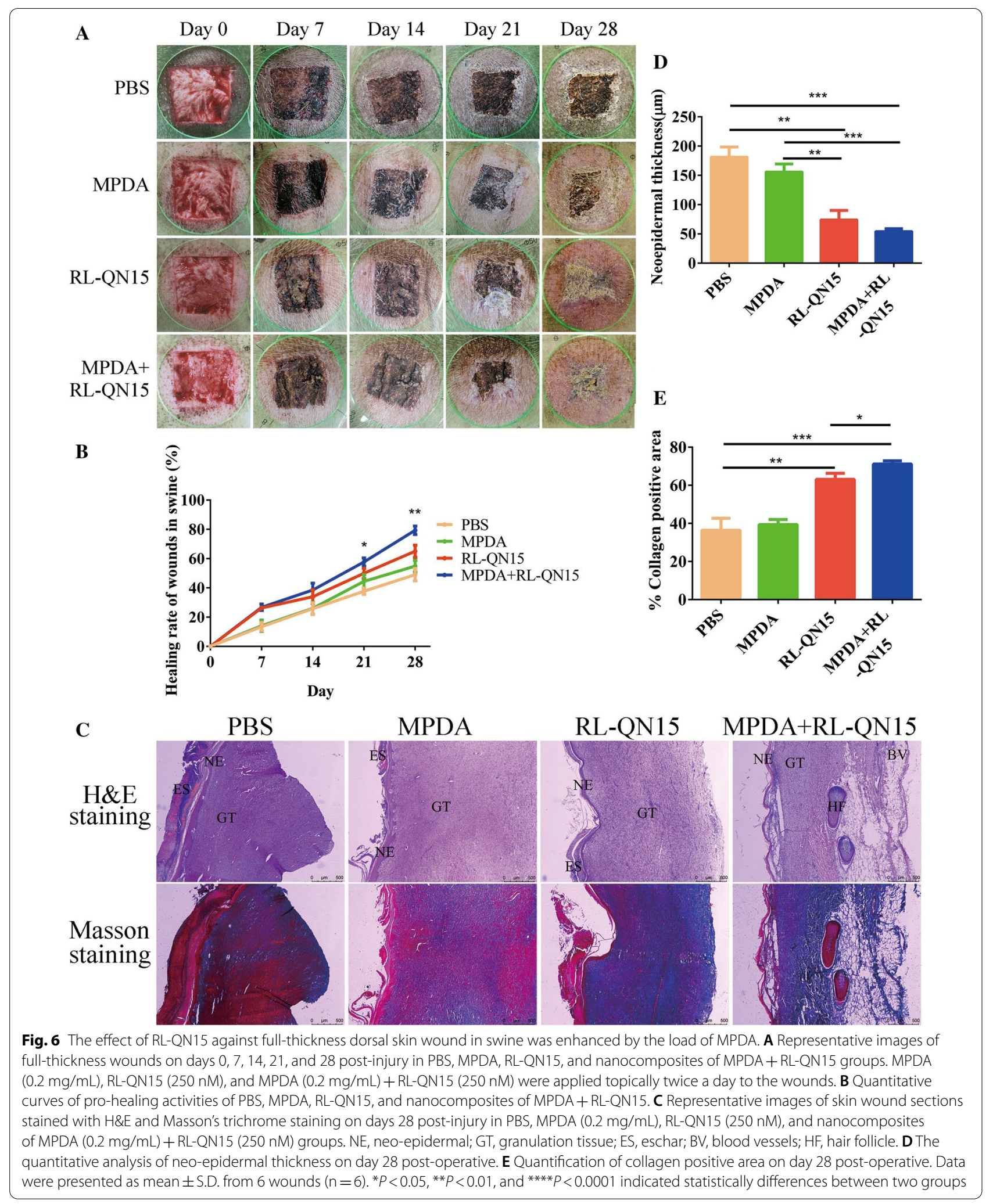


in RL-QN15 and MPDA+RL-QN15 groups was $74.25 \pm 15.89 \mu \mathrm{m}, 54.46 \pm 4.33 \mu \mathrm{m} \quad(\mathrm{n}=6)$ respectively, which was significantly lower than that in PBS group $(181.52 \pm 17.83 \mu \mathrm{m})$ and MPDA group $(156.14 \pm 14.81 \mu \mathrm{m})(\mathrm{n}=6) \quad$ (Fig. 6D). These results show that RL-QN15 not only accelerated wound healing, but also effectively improved the increase in skin thickness, which is consistent with our previous research [23]. In addition, we also quantified the proportion of collagen. There was no significant difference in the proportion of collagen between PBS and MPDA groups $(36.39 \pm 6.78 \%$ vs. $39.38 \pm 2.84 \%(\mathrm{n}=6))$ and the proportion of collagen in RL-QN15 was about $63.11 \pm 3.22 \%(n=6)$, which was significantly higher than that in PBS and PDA groups, but still lower than that in MPDA+RL-QN15 group $(71.13 \pm 1.78 \%)$ $(\mathrm{n}=6)$ (Fig. 6E). In a word, as a carrier of RL-QN15, MPDA successfully improved the prohealing strength of RL-QN15.

\section{The construction of nanocomposite of MPDA} and prohealing peptide might be a promising option for the development of novel prohealing interventions At present, the available intervention for wound repair mainly include various growth factors, small molecular compounds, wound dressings and hydrogels, which are far from meeting the clinical needs, thus the development of novel wound healing therapy is urgently highlighted [55]. In our study, the peptide RL-QN15

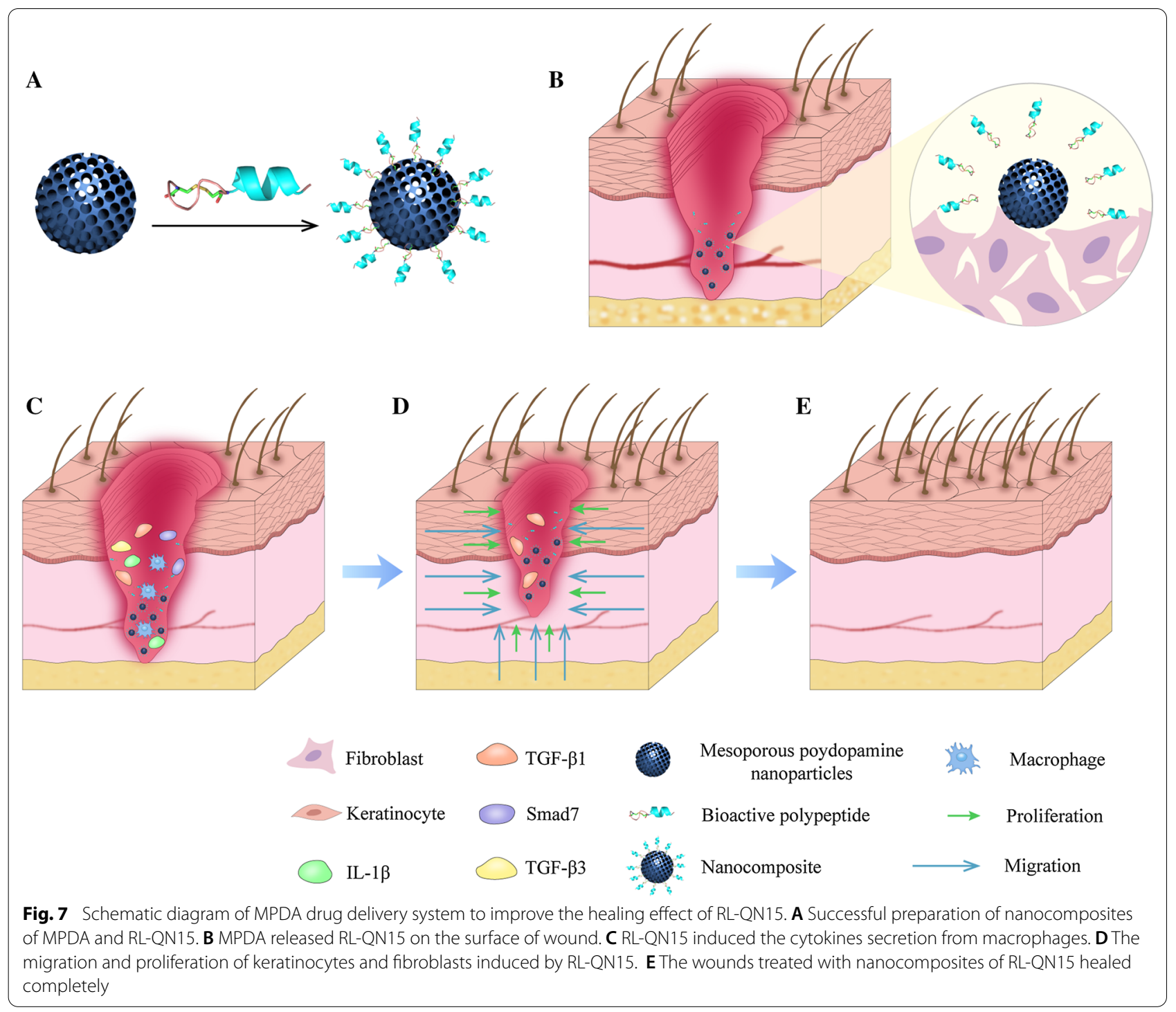


secreted from the skin of R. limnocharis frogs was loaded onto MPDA particles (Fig. 7A). The MPDA particles as drug delivery system loaded with peptides adhere to the wound surface and release RL-QN15 (Fig. 7B). Then, according to our previous research, during the inflammatory phase, RL-QN15 stimulates inflammatory cells, especially macrophages, to secrete cytokines such as TGF- $\beta 1$ [23] (Fig. $7 \mathrm{C}$ ). Which is a multifunctional growth factor that exerts pleiotropic effects on wound healing by regulating cell proliferation and migration, differentiation, ECM production, and immune modulation [56]. Next, the wound enters the proliferative phase. TGF- $\beta 1$ and RL-QN15 promote the proliferation and migration of skin fibroblasts and keratinocytes, resulting in wound closure (Fig. 7D). Finally, during the tissue remodeling period, the skin in the wound site is fully restored, including primarily restoration of the epidermal and dermal layers with appendages, such as hair follicles (Fig. 7E).

Many nanoparticles have been found to promote wound healing through their intrinsic characteristics (antibacterial, anti-oxidant, promote cell proliferation and migration). For example, cerium oxide nanoparticles accelerate the healing of full-thickness dermal wounds in mice via enhancement of the proliferation and migration of fibroblasts, keratinocytes [57]. In addition, compared to porous beads, some functional nanofiber-based scaffolds and antimicrobial dressings are gradually being investigated and applied as an effective strategy for therapeutic wound healing [58-61]. MPDA nanoparticles showed no healing ability, but improved the healing effect of RL-QN15. On the one hand, MPDA nanospheres prolonged the effective concentration of RL-QN15 on the wound surface by continuously releasing RL-QN15. On the other hand, the MPDA microsphere shell protected RL-QN15 from modification by endogenous (e.g., tyrosinase, elastase, metalloproteinase) and exogenous (e.g., produced by colonized microorganisms) enzymes, thus enhancing the therapeutic effect. Therefore, the construction of nanocomposite of MPDA and prohealing peptide might be an option for the development of novel prohealing interventions.

\section{Conclusions}

In the current study, the MPDA nanoparticles and nanocomposites of MPDA and RL-QN15 were successfully prepared. Our results indicated that, compared with RL-QN15 alone, nanocomposites of MPDA and RL-QN15 showed significant increases in the prohealing potency in the full-thickness injured wounds (mice and swine) and burn wounds (mice). Based on our knowledge, this is the first research to report that the load of
MPDA nanoparticles could significantly increase the prohealing potency of peptide and hence highlighted the nanocomposites of MPDA and RL-QN15 as a promising intervention for the acceleration of skin wounds.

\section{Supplementary Information}

The online version contains supplementary material available at https://doi. org/10.1186/s12951-021-01051-8.

Additional file 1: Figure S1. Infrared spectra between $1800 \mathrm{~cm}$ -

1-400 cm-1. Table $\mathbf{S 1}$. Surface area, pore volume and pore diameter of MPDA nanoparticles.

\section{Acknowledgements}

This work was supported by grants from the National Natural Science Foundation of China (81760648, 32060212, 51473089, and 82160159), Yunnan Applied Basic Research Project Foundation (2019FB128), Yunnan Applied Basic Research Project Foundation-Kunming Medical University Union Foundation (2019FE001 (-019), Program for Science \& Technology Innovation Team of Shaanxi Province (No. 2018TD-030), Innovation Capability Support Program of Shaanxi (No.2020TD-024), Fundamental Research Funds for the Central Universities (GK202002007), and Endocrine Clinical Medical Center (ZX2019-02-02), and Diabetic innovation Team (2019HC002) of Yunnan Province.

\section{Authors' contributions}

PQ: Performed the animal experiments, Date curation, Writing-Original draft. YM: Proposed research ideas and design research schemes, Performed the animal experiments. ZD, XG: Synthesized and characterized properties of the MPDA nanoparticles. SY, NL and YH: Helped to conduct the in vivo animal experiments. ZF, YW and HS: Helped to perform the in vitro assays. YW, XY, JT, YY, ZD and XL: Project administration, Funding acquisition. The whole article directed by professor $X Y$ and $Z D$. All authors read and approved the final manuscript.

\section{Availability of data and materials}

The datasets used and/or analyzed during the current study are available from the corresponding author on reasonable request.

\section{Declarations}

Ethics approval and consent to participate

All animal experimental procedures followed the Guide for the care and Use of Laboratory Animals and were approved by the animal ethics committee of Kunming Medical University, China (kmmu20211351).

\section{Consent for publication}

All authors read and approved the fnal manuscript for publication.

\section{Competing interests}

The authors declare no conflict of interest.

\section{Author details}

${ }^{1}$ Department of Anatomy and Histology and Embryology, Faculty of Basic Medical Science, Kunming Medical University, Kunming, Yunnan 650500, China. ${ }^{2}$ Key Laboratory of Applied Surface and Colloid Chemistry, National Ministry of Education, Shaanxi Key Laboratory for Advanced Energy Devices, Shaanxi Engineering Lab for Advanced Energy Technology, School of Materials Science and Engineering, Shaanxi Normal University, Xi'an, Shaanxi 710119 , China. ${ }^{3}$ Key Laboratory of Chemistry in Ethnic Medicine Resource, State Ethnic Affairs Commission and Ministry of Education, School of Ethno-Medicine and Ethno-Pharmacy, Yunnan Minzu University, Kunming, Yunnan 650504, China. ${ }^{4}$ Department of Biochemistry and Molecular Biology, Faculty of Basic Medical Science, Kunming Medical University, Kunming, Yunnan 650500, China. ${ }^{5}$ Department of Endocrinology and Metabolism, Second People's Hospital of Yunnan Province and Affiliated Hospital of Yunnan University, Kunming, Yunnan 650021, China. 
Received: 20 April 2021 Accepted: 20 September 2021

Published online: 09 October 2021

\section{References}

1. Schultz GS, Davidson JM, Kirsner RS, Bornstein P, Herman IM. Dynamic reciprocity in the wound microenvironment. Wound Repair Regen. 2011:19:134-48

2. Landén NX, Li D, Ståhle M. Transition from inflammation to proliferation: a critical step during wound healing. Cell Mol Life Sci. 2016:73:3861-85.

3. Sen CK, Gordillo GM, Roy S, Kirsner R, Lambert L, Hunt TK, Gottrup F, Gurtner GC, Longaker MT. Human skin wounds: a major and snowballing threat to public health and the economy. Wound Repair Regen. 2009; 17:763-71

4. Martin P, Leibovich SJ. Inflammatory cells during wound repair: the good, the bad and the ugly. Trends Cell Biol. 2005;15:599-607.

5. Thomas Hess C. Checklist for factors affecting wound healing. Adv Skin Wound Care. 2011;24:192.

6. Hardwicke J, Schmaljohann D, Boyce D, Thomas D. Epidermal growth factor therapy and wound healing - past, present and future perspectives. Surgeon. 2008:6:172-7.

7. Mascré G, Dekoninck S, Drogat B, Youssef KK, Broheé S, Sotiropoulou PA, Simons BD, Blanpain C. Distinct contribution of stem and progenitor cells to epidermal maintenance. Nature. 2012;489:257-62.

8. Walter MN, Wright KT, Fuller HR, MacNeil S, Johnson WE. Mesenchymal stem cell-conditioned medium accelerates skin wound healing: an in vitro study of fibroblast and keratinocyte scratch assays. Exp Cell Res. 2010:316:1271-81.

9. Nie C, Yang D, Xu J, Si Z, Jin X, Zhang J. Locally administered adiposederived stem cells accelerate wound healing through differentiation and vasculogenesis. Cell Transplant. 2011;20:205-16.

10. Freyman T, Polin G, Osman H, Crary J, Lu M, Cheng L, Palasis M, Wilensky RL. A quantitative, randomized study evaluating three methods of mesenchymal stem cell delivery following myocardial infarction. Eur Heart J. 2006;27:1114-22

11. Khanna S, Biswas S, Shang Y, Collard E, Azad A, Kauh C, Bhasker V, Gordillo GM, Sen CK, Roy S. Macrophage dysfunction impairs resolution of inflammation in the wounds of diabetic mice. PLoS One. 2010;5:e9539.

12. Wang W, Lu KJ, Yu CH, Huang QL, Du YZ. Nano-drug delivery systems in wound treatment and skin regeneration. J Nanobiotechnol. 2019;17:82.

13. Lynge ME, van der Westen R, Postma A, Städler B. Polydopamine-a nature-inspired polymer coating for biomedical science. Nanoscale. 2011:3:4916-28.

14. Liu Y, Ai K, Lu L. Polydopamine and its derivative materials: synthesis and promising applications in energy, environmental, and biomedical fields. Chem Rev. 2014;114:5057-115.

15. Xing Y, Zhang J, Chen F, Liu J, Cai K. Mesoporous polydopamine nanoparticles with co-delivery function for overcoming multidrug resistance via synergistic chemo-photothermal therapy. Nanoscale. 2017;9:8781-90.

16. Zhang Y. Why do we study animal toxins? Dongwuxue Yanjiu. 2015;36:183-222.

17. Musale V, Casciaro B, Mangoni ML, Abdel-Wahab YHA, Flatt PR, Conlon $J M$. Assessment of the potential of temporin peptides from the frog Rana temporaria (Ranidae) as anti-diabetic agents. J Pept Sci. 2018;24.

18. Zhang Y, Wang QQ, Zhao Z, Deng CJ. Animal secretory endolysosome channel discovery. Zool Res. 2021:42:141-52.

19. Wang Y, Cao X, Fu Z, Wang S, Li X, Liu N, Feng Z, Yang M, Tang J, Yang X. Identification and characterization of a novel gene-encoded antioxidant peptide obtained from amphibian skin secretions. Nat Prod Res. 2020;34:754-8.

20. Tang W, Zhang H, Wang L, Qian H, Qi X. Targeted separation of antibacterial peptide from protein hydrolysate of anchovy cooking wastewater by equilibrium dialysis. Food Chem. 2015;168:115-23.

21. Liu N, Wang Y, Yang M, Bian W, Zeng L, Yin S, Xiong Z, Hu Y, Wang S, Meng $B$, et al. New rice-derived short peptide potently alleviated hyperuricemia induced by potassium oxonate in rats. J Agric Food Chem. 2019;67:220-8.

22. Song Y, Wu C, Zhang X, Bian W, Liu N, Yin S, Yang M, Luo M, Tang J, Yang X. A short peptide potentially promotes the healing of skin wound. Biosci Rep. 2019;39.
23. Wang $Y$, Feng $Z$, Yang $M$, Zeng $L$, Qi B, Yin S, Li B, Li Y, Fu Z, Shu L, et al. Discovery of a novel short peptide with efficacy in accelerating the healing of skin wounds. Pharmacol Res. 2020:2020:105296.

24. Xie X, Tang J, Xing Y, Wang Z, Ding T, Zhang J, Cai K. Intervention of Polydopamine Assembly and Adhesion on Nanoscale Interfaces: Stateof-the-Art Designs and Biomedical Applications. Adv Healthc Mater. 2021;2021:e2002138.

25. Guan BY, Yu L, Lou XW. Formation of asymmetric bowl-like mesoporous particles via emulsion-induced interface anisotropic assembly. J Am Chem Soc. 2016:138:11306-11.

26. Dinescu S, Ignat SR, Lazar AD, Marin Ș, Danilă E, Marin MM, Udeanu DI, Ghica MV, Albu-Kaya MG, Costache M. Efficiency of multiparticulate delivery systems loaded with flufenamic acid designed for burn wound healing applications. J Immunol Res. 2019;2019:4513108.

27. Bian W, Meng B, Li X, Wang S, Cao X, Liu N, Yang M, Tang J, Wang Y, Yang X. OA-GL21, a novel bioactive peptide from Odorrana andersonii, accelerated the healing of skin wounds. Biosci Rep. 2018;38.

28. XiY, Ge J, Guo Y, Lei B, Ma PX. Biomimetic elastomeric polypeptidebased nanofibrous matrix for overcoming multidrug-resistant bacteria and enhancing full-thickness wound healing/skin regeneration. ACS Nano. 2018;12:10772-84.

29. Ma H, Zhou Q, Chang J, Wu C. Grape seed-inspired smart hydrogel scaffolds for melanoma therapy and wound healing. ACS Nano. 2019;13:4302-11.

30. Deng Z, Shang B, Peng B. Polydopamine based colloidal materials: synthesis and applications. Chem Rec. 2018;18:410-32.

31. Wang Y, Song S, Lu T, Cheng Y, Song Y, Wang S, Tan F, Li J, Li N. Oxygensupplementing mesoporous polydopamine nanosponges with WS2 QDs-embedded for CT/MSOT/MR imaging and thermoradiotherapy of hypoxic cancer. Biomaterials. 2019;220:119405.

32. Yang $X$, Wang $Y$, Wu $C$, Ling EA. Animal venom peptides as a treasure trove for new therapeutics against neurodegenerative disorders. Curr Med Chem. 2019:26:4749-74.

33. Yang $X$, Lee $W H$, Zhang $Y$. Extremely abundant antimicrobial peptides existed in the skins of nine kinds of Chinese odorous frogs. J Proteome Res. 2012:11:306-19.

34. Guimarães $A B$, Costa FJ, Pires OR, Fontes W, Castro MS. The amazing world of peptide engineering: the example of antimicrobial peptides from frogs and their analogues. Protein Pept Lett. 2016;23:722-37.

35. Wu J, Yang J, Wang X, Wei L, Mi K, Shen Y, Liu T, Yang H, Mu L. A frog cathelicidin peptide effectively promotes cutaneous wound healing in mice. Biochem J. 2018:475:2785-99.

36. Cao X, Wang Y, Wu C, Li X, Fu Z, Yang M, Bian W, Wang S, Song Y, Tang J, Yang $X$. Cathelicidin-OA1, a novel antioxidant peptide identified from an amphibian, accelerates skin wound healing. Sci Rep. 2018;8:943.

37. He X, Yang Y, Mu L, Zhou Y, Chen Y, Wu J, Wang Y, Yang H, Li M, Xu W, Wei $L$. A frog-derived immunomodulatory peptide promotes cutaneous wound healing by regulating cellular response. Front Immunol. 2019:10:2421.

38. Mu L, Tang J, Liu H, Shen C, Rong M, Zhang Z, Lai R. A potential wound-healing-promoting peptide from salamander skin. FASEB J. 2014;28:3919-29.

39. Li X, Wang Y, Zou Z, Yang M, Wu C, Su Y, Tang J, Yang X. OM-LV20, a novel peptide from odorous frog skin, accelerates wound healing in vitro and in vivo. Chem Biol Drug Des. 2018:91:126-36.

40. Easmin S, Sarker MZI, Ghafoor K, Ferdosh S, Jaffri J, Ali ME, Mirhosseini $\mathrm{H}$, Al-Juhaimi FY, Perumal V, Khatib A. Rapid investigation of a-glucosidase inhibitory activity of Phaleria macrocarpa extracts using FTIR-ATR based fingerprinting. J Food Drug Anal. 2017;25:306-15.

41. Ma YR, Zhang XL, Zeng T, Cao D, Zhou Z, Li WH, Niu H, Cai YQ. Polydopamine-coated magnetic nanoparticles for enrichment and direct detection of small molecule pollutants coupled with MALDI-TOF-MS. ACS Appl Mater Interfaces. 2013;5:1024-30.

42. Zhu LP, Jiang JH, Zhu BK, Xu YY. Immobilization of bovine serum albumin onto porous polyethylene membranes using strongly attached polydopamine as a spacer. Colloids Surf B Biointerfaces. 2011:86:111-8.

43. Liu M, Liu T, Zhang X, Jian Z, Xia H, Yang J, Hu X, Xing M, Luo G, Wu J. Fabrication of KR-12 peptide-containing hyaluronic acid immobilized fibrous eggshell membrane effectively kills multi-drug-resistant bacteria, promotes angiogenesis and accelerates re-epithelialization. Int J Nanomed. 2019;14:3345-60. 
44. Wang Y, Beekman J, Hew J, Jackson S, Issler-Fisher AC, Parungao R, Lajevardi SS, Li Z, Maitz PKM. Burn injury: challenges and advances in burn wound healing, infection, pain and scarring. Adv Drug Deliv Rev. 2018;123:3-17.

45. Ipaktchi K, Mattar A, Niederbichler AD, Hoesel LM, Vollmannshauser S, Hemmila MR, Su GL, Remick DG, Wang SC, Arbabi S. Attenuating burn wound inflammatory signaling reduces systemic inflammation and acute lung injury. J Immunol. 2006;177:8065-71.

46. Bae SH, Bae YC, Nam SB, Choi SJ. A skin fixation method for decreasing the influence of wound contraction on wound healing in a rat model. Arch Plast Surg. 2012;39:457-62.

47. Cai EZ, Ang CH, Raju A, Tan KB, Hing EC, Loo Y, Wong YC, Lee H, Lim J, Moochhala SM, et al. Creation of consistent burn wounds: a rat model. Arch Plast Surg. 2014;41:317-24.

48. Davidson JM. Animal models for wound repair. Arch Dermatol Res. 1998;290(Suppl):1-11.

49. Sullivan TP, Eaglstein WH, Davis SC, Mertz P. The pig as a model for human wound healing. Wound Repair Regen. 2001;9:66-76.

50. Lee PY, Park SG, Kim EY, Lee MS, Chung SJ, Lee SC, Yu DY, Bae KH. Proteomic analysis of pancreata from mini-pigs treated with streptozotocin as a type I diabetes models. J Microbiol Biotechnol. 2010;20:817-20.

51. Fricker M, Deane A, Hansbro PM. Animal models of chronic obstructive pulmonary disease. Expert Opin Drug Discov. 2014;9:629-45.

52. Meurens F, Summerfield A, Nauwynck H, Saif L, Gerdts V. The pig: a model for human infectious diseases. Trends Microbiol. 2012;20:50-7.

53. Lindblad WJ. Considerations for selecting the correct animal model for dermal wound-healing studies. J Biomater Sci Polym Ed. 2008;19:1087-96.

54. Wollina U, Berger U, Mahrle G. Immunohistochemistry of porcine skin Acta Histochem. 1991;90:87-91.
55. Das S, Baker AB. Biomaterials and nanotherapeutics for enhancing skin wound healing. Front Bioeng Biotechnol. 2016;4:82.

56. Penn JW, Grobbelaar AO, Rolfe KJ. The role of the TGF- $\beta$ family in wound healing, burns and scarring: a review. Int J Burns Trauma. 2012;2:18-28.

57. Chigurupati S, Mughal MR, Okun E, Das S, Kumar A, McCaffery M, Seal S, Mattson MP. Effects of cerium oxide nanoparticles on the growth of keratinocytes, fibroblasts and vascular endothelial cells in cutaneous wound healing. Biomaterials. 2013;34:2194-201.

58. Chen CK, Liao MG, Wu YL, Fang ZY, Chen JA. Preparation of highly swelling/antibacterial cross-linked N-maleoyl-functional chitosan/polyethylene oxide nanofiber meshes for controlled antibiotic release. Mol Pharm. 2020;17:3461-76.

59. Su Y, Wang H, Mishra B, Lakshmaiah Narayana J, Jiang J, Reilly DA, Hollins RR, Carlson MA, Wang G, Xie J. Nanofiber dressings topically delivering molecularly engineered human cathelicidin peptides for the treatment of biofilms in chronic wounds. Mol Pharm. 2019;16:2011-20.

60. Chen CK, Lee MC, Lin ZI, Lee CA, Tung YC, Lou CW, Law WC, Chen NT, Lin KA, Lin JH. Intensifying the antimicrobial activity of poly[2-(tertbutylamino)ethyl methacrylate]/polylactide composites by tailoring their chemical and physical structures. Mol Pharm. 2019;16:709-23.

61. Rieger KA, Birch NP, Schiffman JD. Designing electrospun nanofiber mats to promote wound healing — a review. J Mater Chem B. 2013;1:4531-41.

\section{Publisher's Note}

Springer Nature remains neutral with regard to jurisdictional claims in published maps and institutional affiliations.
Ready to submit your research? Choose BMC and benefit from:

- fast, convenient online submission

- thorough peer review by experienced researchers in your field

- rapid publication on acceptance

- support for research data, including large and complex data types

- gold Open Access which fosters wider collaboration and increased citations

- maximum visibility for your research: over 100M website views per year

At BMC, research is always in progress.

Learn more biomedcentral.com/submissions 